\title{
Prevalência da deficiência de Glicose-6-Fosfato Desidrogenase em uma população adulta
}

\author{
Aline PERINOTO ${ }^{1}$ \\ Carolina Ribeiro de Oliveira Brandão COSTA ${ }^{2}$ \\ Guilherme Silveira DUARTE ${ }^{3}$ \\ Ramon Alves de Oliveira PAULA ${ }^{4}$ \\ Fernanda Borges de Araujo PAULA ${ }^{5}$ \\ Stella Maris da Silveira DUARTE ${ }^{6}$
}

1 Farmacêutica pela Universidade Federal de Alfenas .

2 Graduanda em Farmácia, Universidade Federal de Alfenas.

3 Graduando em Medicina, Universidade José do Rosário Vellano.

4 Graduando em Farmácia, Universidade Federal de Alfenas.

5Farmacêutica. Professora Associada. Universidade Federal de Alfenas.

6 Farmacêutica. Professora Associada. Universidade Federal de Alfenas.

\section{Autor correspondente:}

Prof. Dra. Stella Maris da Silveira Duarte

Departamento de Análises Clínicas e Toxicológicas

Universidade Federal de Alfenas-MG

37130-000 - Alfenas - MG- Brasil

E-mail: stellaunifal@yahoo.com.br

Recebido em: 12/05/2013 - Aprovado em: 15/07/2013 - Disponibilizado em: 15/08/2013

\section{RESUMO}

A glicose-6-fosfato desidrogenase (G6PD) desempenha funções de extrema importância no metabolismo eritrocitário, atuando tanto na obtenção de energia a partir da glicose, quanto na sua proteção contra agentes oxidantes. A deficiência de G6PD é uma eritroenzimopatia bastante comum, sendo um distúrbio de origem hereditária, acometendo principalmente indivíduos do sexo masculino e negróides. A patologia leva a um quadro clínico bastante heterogêneo, sendo sua principal manifestação a anemia hemolítica decorrente da hemólise, devido ao consumo de certas substâncias com caráter oxidante. Esta pesquisa teve como objetivo, determinar a prevalência de portadores da deficiência de glicose-6-fosfato desidrogenase em estudantes do sexo masculino de Alfenas-MG. A fim de uma maior adesão ao projeto e esclarecimento dos interessados, realizaram-se palestras educativas e distribuição de folhetos explicativos. Após este procedimento, as amostras de sangue colhidas por punção venosa em tubos com anticoagulante (EDTA) foram analisadas, depois da autorização dos participantes através da assinatura do termo de concordância. A técnica utilizada foi o teste da redução da metemoglobina (teste de Brewer), sendo este um teste qualitativo para detecção da deficiência. A população estudada constitui-se de 200 indivíduos do sexo masculino, dentre os quais, 6 participantes (3\%) tinham a atividade deficiente da G6PD e 194 apresentavam atividade normal. Os indivíduos identificados com esta doença, receberam o resultado do exame onde foram orientados e encaminhados para o médico.

PALAVRAS-CHAVE: Deficiência de G6PD. Enzimopatia. Anemia hemolítica. Hemólise. Portadores.

\section{Prevalence of glucose-6-phosphate dehydrogenase in an adult population}

\begin{abstract}
Glucose-6-phosphate dehydrogenase (G6PD) plays very important roles in erythrocyte metabolism, acting both in obtaining energy from glucose, and in their protection against oxidizing agents. G6PD deficiency is a very common eritroenzimopatia, being a hereditary disorder, primarily affecting males and Negroid. The disease leads to a clinical heterogeneous, being its main manifestation hemolytic anemia due to hemolysis, due to consumption of certain substances with oxidising character. This study aimed to determine the prevalence of carriers of glucose-6-phosphate dehydrogenase deficiency in male students Alfenas-MG. In order to further clarify and adherence to project stakeholders, there were educational lectures and distributing leaflets. After this procedure,the blood samples collected by venipuncture in tubes with anticoagulant (EDTA) were analyzed, after the authorization of participants through the
\end{abstract}


signing of the agreement. The technique used was the test of the methemoglobin reduction (test Brewer), which is a qualitative test for detection of deficiency. The study population consisted of 200 male subjects, among which 6 participants (3\%) had deficient activity of G6PD and 194 had normal activity. Individuals identified with this disease, received the test results which were targeted and referred to the doctor.

KEYWORDS: G6PD deficiency. Enzymopathy. Hemolytic anemia. Hemolysis. Carriers.

\section{INTRODUÇÃO}

A deficiência de Glicose-6-fosfato desidrogenase (G6PD) é uma doença hereditária, causada por mutações no gene codificante para G6PD localizado no cromossomo $\mathrm{X}$ (lócus Xq28). As manifestações clínicas ocorrem geralmente em homens hemizigotos e em mulheres homozigotas para o cromossomo anormal.

Em todo mundo, esta enzimopatia, afeta por volta de 330 milhões de pessoas, existindo variantes da mesma. Embora a maioria dos indivíduos afetados seja assintomática, o uso de drogas com propriedades oxidantes e o consumo de alimentos que possibilitem formação do grupo sulfidrila (favas) pode levar o portador da deficiência de G6PD a uma crise hemolítica aguda e intensa (ABREU; FREIRE; NKHOMA AT, 2009; MIURA, 2002; KATSURAGAWA et al., 2004).

A G6PD é uma enzima citoplasmática amplamente distribuída entre quase todos os organismos e tecidos. Nos seres humanos, apesar da ampla distribuição, é no metabolismo das hemácias que essa enzima exerce a sua função mais importante, ao atuar em uma das vias metabólicas utilizadas por estas células para obtenção de energia. $\mathrm{Na}$ reação catalisada pela G6PD, é produzido o fosfato reduzido de nicotinamida adenina dinucleotídeo (NADPH), substância redutora essencial à proteção das hemácias contra a ação de agentes oxidantes de origem endógena (peróxidos orgânicos) e exógena (drogas, alimentos e elementos atmosféricos). Assim, a atividade da G6PD é indispensável à manutenção da integridade das hemácias e a acentuada deficiência dessa enzima pode produzir sérias alterações metabólicas, sobretudo na presença de substâncias oxidantes.

A principal consequência dessas alterações metabólicas é a hemólise, determinada fundamentalmente pela precipitação da hemoglobina e formação de corpúsculos de Heinz, pela oxidação dos grupos tiol das enzimas citoplasmáticas e da membrana celular e, secundariamente, pela oxidação de lípides da membrana eritrocitária (COMPRI; SAAD; RAMALHO, 2000; BEUTLER, 1994).

A hemólise pode ser desencadeada por infecções e por algumas drogas com propriedades oxidativas como o ácido acetilsalicílico, a vitamina $\mathrm{K}$, o cloranfenicol e antimaláricos. Além de fatores externos como situações de estresse ou infecções, também podem precipitar crises hemolíticas 
nos casos de deficiência de G6PD. Hepatite alcoólica, hepatite viral, pneumonias, septicemias, acidose diabética e paludismo são alguns exemplos de fatores que podem desencadear hemólise nestes pacientes (ABREU; FREIRE; MIURA, 2002).

O diagnóstico desta enzimopatia é relativamente difícil, porque a dosagem da enzima no sangue pode apresentar- se normal durante uma crise hemolítica aguda desencadeada por drogas oxidantes ou estresse. Normalmente a hemólise aparece de um a três dias após o contato com os fatores desencadeantes. (FONSECA et al., 2003).

A prevenção da hemólise é a medida fundamental no manuseio anestésico dos pacientes, evitando-se a administração de drogas oxidantes, a ingestão de determinados alimentos ou situações de estresse, pois as consequências clínicas após um insulto que resulta em hemólise poderão ser extremamente variáveis: desde anemia hemolítica até o óbito, nos casos de hemólise maciça (ABREU; FREIRE; MIURA, 2002; LORENZI et al., 2003).

Diante disto, o presente estudo teve como objetivo determinar a prevalência da deficiência de glicose-6-fosfato desidrogenase em alunos do sexo masculino matriculados na Unifal-MG e de adolescentes do Tiro de Guerra de Alfenas-MG.

\section{MATERIAIS E MÉTODOS}

No período de maio de 2011 a abril de 2012 foram analisadas amostras de sangue de
200 estudantes do sexo masculino que aceitaram participar deste estudo através da assinatura do Termo de Consentimento Livre e Esclarecido (TCLE) conforme preconiza a Resolução 196/96 do Conselho Nacional de Saúde.

O trabalho foi realizado em duas etapas, como detalhado abaixo.

Primeira etapa: Inicialmente, foram oferecidas palestras educativas visando conscientizar os voluntários da importância do diagnóstico da deficiência da Glicose - 6 Fosfato Desidrogenase.

As palestras foram ministras ao público alvo utilizando-se aparelho multimídia como recurso didático. Utilizou-se uma linguagem clara e objetiva, porém sem perda do caráter científico, respeitando a autonomia dos participantes em se ausentarem do local, a qualquer momento, sem necessidade de apresentar justificativa aos pesquisadores. Foram distribuídos folders ilustrativos.

Logo após as palestras e os esclarecimentos de eventuais dúvidas por parte do público alvo, foram oferecidos a realização de exames laboratoriais sendo estes o eritrograma e a determinação da atividade da Glicose- 6 -Fosfato Desidrogenase. Só realizaram os exames os indivíduos que concordaram com o TCLE

A coleta das amostras foi realizada mediante punção venosa e o sangue colocado em tubos contendo anticoagulante (EDTA) na proporção de $1 \mathrm{mg} / \mathrm{mL}$ de sangue. Todas as 
amostras foram submetidas à determinação da atividade da G6PD. As amostras deficientes foram confirmadas através do teste quantitativo de redução da metahemoglobina proposto por Beutler (1984) e pesquisa de corpúsculo de Heinz após coloração com azul de cresil brilhante de acordo com Carvalho (1994).

Segunda etapa: Ocorreu a entrega dos resultados dos exames laboratoriais aos participantes. Os indivíduos com resultados alterados foram orientados e encaminhados ao médico.

As pesquisadoras responsáveis pela execução do trabalho e pela obtenção do TCLE assinado pelo paciente e/ou responsável legal são acadêmicas do Curso de Farmácia, sempre supervisionadas pelo professor orientador.

Os resultados dos exames foram mantidos em sigilo e entregues aos participantes com orientação e, em caso positivo, encaminhados para um médico.

Esta pesquisa teve aprovação do Comitê de Ética em Pesquisa em Seres Humanos da Universidade Federal de Alfenas (Protocolo de Pesquisa n ${ }^{\circ}$ 038/2011).

\section{RESULTADOS E DISCUSSÃO}

A análise das amostras pelo teste qualitativo da redução da metemoglobina empregada demonstrou que 6 indivíduos tinham a atividade de G6PD deficiente e 194 apresentavam atividade normal. Os pacientes positivos para esta eritroenzimopatia foram notificados, orientados e encaminhados para o médico após terem recebido o resultado do exame.

Sabe-se que a prevalência global da deficiência de Glicose-6-fosfato desidrogenase, encontra-se geograficamente correlacionada às áreas endêmicas em malária, sendo maiores na África SubSahariana, Oriente Médio, Sudeste da Ásia, Europa Mediterrânea e algumas áreas da América Latina (Nkhoma A.T. et. al., 2009). Em algumas regiões da África a deficiência de G6PD é encontrada em $26 \%$ da população (BEZERRA, ALBULQUERQUE, LIMA, LINS, 1990; KATSURAGAWA, 2004).

Estudos realizados na população brasileira revelaram uma prevalência de deficientes de G6PD em torno de $1 \%$ a $10 \%$, com os maiores índices sendo encontrados entre os homens de ascendência africana (Silva et al., 2005). A prevalência obtida na população estudada foi de $3 \%$, sendo compatível com outros estudos realizados no Brasil. A mesma proporção, foi encontrada em estudos conduzidos por Katsuragawa, (2004). Considerando este índice, podemos afirmar que o Brasil possui 6 milhões de pessoas deficientes de G6PD (LEITE, 2010). Maurício et al (2006) mostraram que $3,5 \%$ da amostra de 569 pacientes atendidos em hospital de referência da cidade de Natal, foram notificados com esta patologia. Resultados semelhantes foram identificados em outro estudo onde $27(3,8 \%)$ dos 714 indivíduos, apresentaram deficiência da 
G6PD. Dentre os deficientes, 21 (77,8\%) eram do sexo masculino e $6(22,2 \%)$ eram do sexo feminino. (FERNANDES et al ; 2010).

A identificação dos portadores da deficiência de G6PD é extremamente importante, uma vez que estes podem manifestar uma crise hemolítica aguda de intensidade variável na presença de agentes oxidantes de origem endógena ou exógena, podendo ser gerada por fármacos (sulfas e sulfonas, antimaláricos, nitrofuranos e vários analgésicos e antipiréticos, como a acetanilida e a aspirina) e por alimentos (leguminosa Vicea fava, enlatados ricos em nitritos) (BEZERRA, ALBULQUERQUE, LIMA, LINS, 1990; KATSURAGAWA, 2004).

Em um estudo onde avaliou-se a incidência da deficiência de Glicose-6-Fosfato Desidrogenase (G6PD) e perfil hematológico em indivíduos de uma região de Rondônia, foram analisadas amostras de 122 indivíduos, sendo 69 homens e 53 mulheres, com idades entre 3 a 84 anos. Quatro casos $(5,8 \%)$ do sexo masculino apresentaram resultado positivo para deficiência da G6PD enquanto nenhuma ocorrência foi verificada na população feminina (KATSURAGAWA et al., 2004). O estudo de Iglessias et al (2010), demonstra que $3,0 \%$ dos recém-nascidos do sexo masculino pesquisados são deficientes de G6PD.

Pesquisa conduzida por Santos (2005) demonstraram que das 297 amostras de pacientes com diagnóstico de HIV positivo, $150(50,5 \%)$ indivíduos eram do sexo masculino e 147 (49,5\%) do sexo feminino. Dentre a população estudada, quatro pacientes apresentaram deficiência parcial de G6PD, correspondendo a uma prevalência de 1,3\%. A autora observou baixa prevalência quando comparada aos resultados encontrados por Silva et al (2006). Isto se deve, provavelmente pelo menor número de afrodescendentes na amostra analisada, representando pacientes de baixo risco para desenvolver crises hemolíticas.

Medeiros et al (2006) avaliaram a prevalência de deficiência de Glicose- 6 Fosfato Desidrogenase em pacientes atendidos no Hospital Universitário Onofre Lopes, de Natal. Dos 569 pacientes, foram identificados 20 deficientes de G6PD correspondendo a uma prevalência de 3,5\%, sendo $1,6 \%$ e $4,3 \%$ entre os brancos e pardos, respectivamente.

Resultados opostos foram encontrados no estudo de Acosta et al (2012) que realizaram pesquisa da enzima G6PD em uma população de homens e mulheres na cidade de Rosário, Argentina, onde encontraram dois pacientes $(0,3 \%)$ portadores de deficiência de G6PD, do total de 686 participantes. Mañú Pereyra $\mathrm{M} \operatorname{del} \mathrm{M}$ et al., (2007) encontraram em um estudo desenvolvido na Espanha, prevalência de 0,1 a $0,5 \%$.

Os indivíduos portadores da eritroenzimopatia geralmente são assintomáticos, entretanto, eles podem manifestar hemólise de graus variáveis 
quando em contato com alguns fármacos ou quando expostos a uma condição de estresse oxidativo (BEZERRA, ALBULQUERQUE, LIMA， LINS， 1990; KATSURAGAWA, 2004).

\section{CONCLUSÃO}

Diante do exposto conclui-se que é de suma importância a triagem de indivíduos portadores da G6PD considerando a gravidade das manifestações clínicas de forma a contribuir para o esclarecimento sobre a

\section{REFERÊNCIAS BIBLIOGRÁFICAS}

ABREU, M. P.; FREIRE, C. C. S.; MIURA,

R. S. Anestesia em paciente portador de deficiência de Glicose-6-Fosfato

Desidrogenase. Rev. Bras. Anestesiol. Campinas, v. 6, n. 52, p. 707-711, 2002.

\section{ACOSTA I del L et al. Deficiencia de} glucosa-6-fosfato deshidrogenasa eritrocitaria en Rosario. Acta Bioquím Clín Latinoam 2012; 46 (3): 359-63

ATAY, E.; BOZAYKUT A.; IPEK I.

Glucose-6-phosphate dehydrogenase deficiency in neonatal indirect hyperbilirrubinemia. Journal of Tropical

Pediatrics, v. 1, n. 52, p. 56-58, 2006.

BAIN, B. J. Células sanguíneas. 2. ed. Porto Alegre: Artes Médicas, 1997. doença e uma orientação profilática. Vale ressaltar também que são escassas as referências na literatura que tratam a respeito da deficiência dessa enzima, sendo necessários mais trabalhos comunitários. sistematizados, tendo em vista sua alta prevalência.

\section{AGRADECIMENTOS}

A Unifal-MG; ao PROEXT/MEC pelo apoio concedido.

BARRETTO, O. C. O. P. Metabolismo do glóbulo vermelho: revisão. Arq. Med., ABC, n. 1, p. 17-21, 1978.

BATISTA FILHO, M. O controle das anemias no Brasil. Rev. Bas. Saúde Materno Infantil, v. 2, n. 4, p. 121-123, 2004.

BERHMAN, R. E. N. Doenças do sangue: anemia hemolítica por drogas: tratado de pediatria, 14. ed. Rio de Janeiro: Guanabara Koogan, v. 2, n. 1094, 1994.

BEUTLER, E. G6PD deficiency. Blood, New York, v. 84, n. 11, p. 3613-3636, 1994.

BEZERRA, T.M.M.; ALBULQUERQUE, L.M.M.; LIMA, A.A.B.; LINS, M.R.S. Deficiência de glicose-6-fosfato 
desidrogenase em adultos jovens. Rev. Bras.

Anal.Clin., v. 22, n. 4, p. 91-92, 1990.

BORGES, A. R. et al. Deficiência da glicose-

6-fosfato desidrogenase

com infeções de repetição: relato de caso.

Jornal de Pediatria, Rio de Janeiro, v. 4, n.

77, p. 331-336, 2001.

BREWER, G. J.; TARLOV, A. R.; ALVING,

A. S. The methemoglobin reduction test for primaquine -type sensitivity of erythrocytes.

Journal of the American Medical

Association, New York, n. 180, p. 386-388, 1962.

CARVALHO, W. F. Técnicas médicas de

hematologia e imunohematologia. 6. ed.

Belo Horizonte: Coopmed, 1994.

CIANCIARULLU, M. A. et al. Perda

auditiva neonatal associada a

hiperbilirrubinemia por deficiência de glicose-

6-fosfato desidrogenase: relato de caso.

Pediatria, v. 2, n.27, p. 126-132, 2005.

COMPRI, M. B.; SAAD, S. T. O.;

RAMALHO, A. S. Investigação genético-

epidemiológica e molecular da deficiência de

G6PD em uma comunidade brasileira. Cad.

Saúde Pública, Rio de Janeiro, v. 16, n.

2, 2000 .

FERNANDES et al., 2010. Prevalência da

deficiência da glicose- 6-fosfato desidrogenase em doadores de sangue de Mossoró, Rio Grande do Norte. Rev Bras

\section{Hematologia e Hemoterapia.}

2010;32(5):422-423

FONSECA, F. L. A. et al. A utilização do laboratório clínico na investigação das eritroenzimopatias. 2003. Disponível em: $<$ http://www3.fsa.br/proppex/radar/Artigos\% 20em\%20PDF/Eritroenzimopatias.pdf $>$. Acesso em: 15 maio 2010.

GARLIPP, C.R.; RAMALHO, A.S; Aspectos clínicos e laboratoriais da deficiência de desidrogenase de 6-fosfato de glucose (G6PD) em recém nascidos brasileiros. Rev.

Bras. Genet. 11(3): 717-728, 1988.

HOFFBRAND, A. V.; PETIT, J. E.; MOSS, P. A. H. Fundamento em hematologia. 4. ed. Oxford: Blackwell Science, 2002.

IGLESSIAS, M. A. C. Frequência da deficiência de glicose-6-fosfato desidrogenase (G6PD) e sua relação com a icterícia neonatal. 2008. Dissertação (mestrando em Ciências Farmacêuticas) UFRGN, Natal, 2008.

IGLESSIAS, M. A.; SANTOS, R. M.; AMORIN, M. S.; SILVA, R. T.;

MOREIRA, S. S.; BARRETTO, O. C.;

MEDEIROS, T. M. Deficiência de glicose-6fosfato desidrogenase eritrocitária em recémnascidos do sexo masculino e sua relação com 
a icterícia neonatal. Rev Bras Hematol

Hemoter. 2010.

KATSURAGAWA, T. H. et al. Avaliação da incidência da deficiência de Glicose-6-Fosfato Desidrogenase (G6PD) e perfil hematológico em indivíduos de uma região de Rondônia.

Rev. bras. hematol. Hemoter., São José do Rio Preto, v. 4, n. 26, p. 268-273, 2004.

LEITE, A.A. Icterícia neonatal e deficiência de glicose-6-fosfato desidrogenase Comentários científicos / Scientific commentaries Rev Bras

Hematol Hemoter. 2010;32(6):430-431.

\section{LORENZI, T. F. et al. Manual de}

hematologia: propedêutica e clínica. 3. ed.

Rio de Janeiro: Medsi, 2003.

LUKENS, J. N. Anemias hemolíticas hereditárias associadas a anormalidades da glicólise anaeróbica dos eritrócitos e metabolismo dos nucleotídeos. Wintrobe Hematologia Clínica., São Paulo, v. 1, p. 1084-1100, 1998.

Mañú Pereyra M del M. et al. Cribado neonatal de hemoglobinopatías y déficit de glucosa-6-fosfato deshidrogenasa (G6PD) en Cataluña. Estudio molecular de la anemia falciforme asociada a alfatalasemia y déficit de G6PD. Med Clin (Barc) 2007; 129(5): 161-4.
MAURÍCIO, C. R. F. et al.; Deficiência de glicose-6-fosfato desidrogenase: dados de prevalência em pacientes atendidos no Hospital Universitário Onofre Lopes. RBAC, v. 1, n.38, p.57-59, 2006.

MCDONALD, G. A.; PAUL, J.; CRUICKSHANK, B. Atlas de hematologia. Madrid: Médica Panamericana, 1995. MIWA, S.; FUJII, H. Molecular basis of erythroenzymopathies associated with hereditary hemolytic anemia: tabulation of mutation enzymes. American Journal of Hematology, v. 151, p. 122-132, 1996.

NAOUM, P. C; NAOUM, F. A. Hematologia

Laboratorial: Eritrócitos. São José do Rio

Preto: Editora Academia de Ciência e

Tecnologia, 2005.

NKHOMA, A. T.; POOLE, C.;

VANNAPPAGARI, V., HALL, A. S., BEUTLER, E. The global prevalence of glucose-6-phosphate dehydrogenase deficiency: a systematic review and metaanalysis. Blood Cells Mol Dis. 2009; 42(3):267-78.

OLIVEIRA, M. A. A.; OSÓRIO, M. M.; RAPOSO, M. C. F. Concentração de hemoglobina e anemia em crianças no Estado de Pernambuco, Brasil: fatores sócioeconômicos e de consumo alimentar associados. Cad. Saúde Pública, v. 10, n. 22, p. 2169-2178, 2006. 
RAMALHO, A.S.; Deficiência de desidrogenase de 6-fosfato de glucose (G6PD) em recém-nascidos brasileiros. Rev.

Assoc. Med. Bras. 27(12):343-345, 1981.

SCHIAR, V. P. P. Avaliação toxicológica de organocalcogênicos em eritrócitos

humanos. 2009. 78f. Tese (Doutorado em

Bioquímica toxicológica) - UFSM, Santa

Maria, 2009.

SILVA, L. H. P.; OLIVEIRA, V. E. G. O desafio da malária: o caso brasileiro e o que se pode esperar dos progressos da era genômica. Ciência e Saúde Coletiva, Rio de Janeiro, v. 7, n. 1, p. 49-63, 2002.

\section{SANTOS, V. F. Prevalência da deficiência}

de glicose-6-fosfato desidrogenase em pacientes HIV soro-positivos. 2005. 10p.

Trabalho de conclusão de curso Biomedicina, Centro Universitário Feevale.

Novo Hamburgo, 2005.

SILVA, M. C. M. et al. Alterações

clínicolaboratoriais em pacientes com malária por Plasmodium vivax e deficiência de glicose-6-fosfato desidrogenase tratados com
0,50 $\mathrm{mg} / \mathrm{kg} /$ dia de primaquina. Revista da Sociedade Brasileira de Medicina Tropical, v. 3, n. 37, p. 215-217, 2004.

SILVA, S. C. L. S.; BATISTA FILHO, M..; MIGLIOLI, T. C. Prevalência e fatores de risco de anemia em mães e filhos no Estado de Pernambuco. Rev. Bras. Epidemiol.,São Paulo, v. 11 n. 2, 2008.

SILVA R.T. et al. Deficiências de glicose-6fosfato desidrogenase em adultos. Newslab [Internet]. 2005 [citado 2009 Jul 27];79:96-

102. Disponível em:

http://www.newslab.com.br/ed_anteriores/79/ art03/art03.pdf

SILVA et al., 2006. Deficiência de Glicose6-Fosfato Desidrogenase em Adultos.

NewsLab - edição 79 - 2006. pag 96 a 102.

ZAGO, M. A. et al. Hematologia

Fundamentos e Prática. 1. ed, São Paulo: Atheneu, 2001. 